\title{
Some Surprising Results on a One-Dimensional Elliptic Boundary Value Blow-Up Problem
}

\author{
Y. J. Cheng
}

Abstract. In this paper we consider the one-dimensional elliptic boundary blow-up problem

$$
\left.\begin{array}{l}
\Delta_{p} u=f(u) \quad(a<t<b) \\
u(a)=u(b)=+\infty
\end{array}\right\}
$$

where $\Delta_{p} u=\left(\left|u^{\prime}(t)\right|^{p-2} u^{\prime}(t)\right)^{\prime}$ is the usual $p$-Laplace operator. We show that the structure of the solutions can be very rich even for a simple function $f$ which gives a leading that a simliar results might hold also in higher dimensional spaces

Keywords: Boundary blow-up, multiplicity, concave and convex nonlinearity

AMS subject classiflcation: $34 \mathrm{~B}, 35 \mathrm{~J}$

\section{Introduction and formulation of main results}

In the last few years there is a great of interests in the investigation of boundary blowup solutions for elliptic equations $[2,3,7]$, which comes originally from differential geometry [6] and electrohydrodynamics [5]. Very recently some existence results of two (one positive and one sign-changing) solutions have been established in $[1,8]$. The purpose of the present paper is to show through one-dimensional examples that the structure of the solutions can be very rich even for a simple right-hand side. More precisely, we consider the problem

$$
\left.\begin{array}{l}
\Delta_{p} u=\lambda f(u) \quad(a<t<b) \\
u(a)=u(b)=+\infty
\end{array}\right\}
$$

where

$$
\Delta_{p} u=\left(\left|u^{\prime}(t)\right|^{p-2} u^{\prime}(t)\right)^{\prime}
$$

is the $p$-Laplace operator as usual, $\lambda>0$ is a parameter, and $f$ is a given continuous function. By a solution $u=u(t)$ of problem (1) we mean that $u$ satisfies the equation in (1), i.e. $\left(\left|u^{\prime}(t)\right|^{p-2} u^{\prime}(t)\right)^{\prime}=\lambda f(u(t))$ for all $t \in(a, b)$, and $\lim _{t \rightarrow a+} u(t)=\lim _{t \rightarrow b-} u(t)=$ $+\infty$. By a sign-changing solution $u(t)$ of (1) we mean that there exist $t_{1}, t_{2} \in(a, b)$ such that $u\left(t_{1}\right)>0$ and $u\left(t_{2}\right)<0$.

The results for problem (1) in this paper are summarized in the followings three theorems.

Yuanji Cheng: Univ. of Malmö, School of Techn. and Management., 20506 Malmö, Sweden

ISSN 0232-2064 / \$2.50 C Heldermann Verlag Berlin 

let

Theorem 1. For given positive constants $q, \varepsilon, s$ and $\alpha, r$ such that $q>p-1>r>s$

$$
f(u)=u^{g}+\varepsilon u^{s} \quad(u \geq 0) \quad \text { and } \quad f(u)=\alpha|u|^{r} \quad(u \leq 0) .
$$

Then the following statements hold:

(i) There exists a constant $\lambda_{1}>0$ such that problem (1) has at least one signchanging solution if $\lambda>\lambda_{1}$, and no such kind of solutions can exist if $\lambda<\lambda_{1}$.

(ii) There exist a constant $\varepsilon_{0}>0$ such that the solution is unique if $\varepsilon \geq \varepsilon_{0}$.

(iii) If $\varepsilon \in\left(0, \varepsilon_{0}\right)$, then there are constants $\lambda_{2}<\lambda_{3} \leq \lambda_{4}$ such that problem (1) has at least two sign-changing solutions if $\lambda \in\left(\lambda_{1}, \lambda_{4}\right)$, has at least three sign-changing solutions if $\lambda \in\left(\lambda_{2}, \lambda_{3}\right)$ ), and has a unique sign-changing solution if $\lambda>\lambda_{4}$.

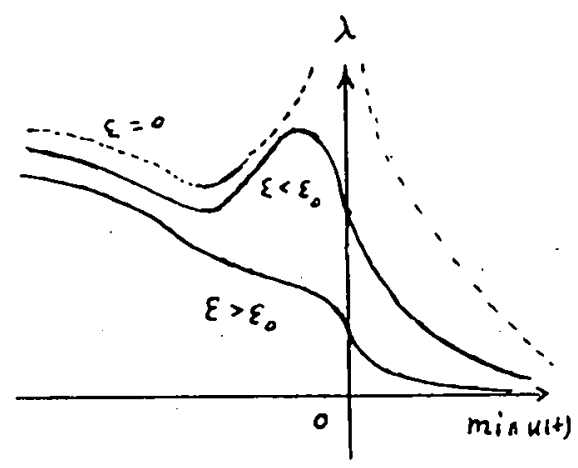

Figure 1

Theorem 2. For given positive constants $q, \varepsilon, s$ and $\alpha, r, \delta, \tau$ such that $q, \tau>p-1>$ $r>s$ let

$$
f(u)=u^{q}+\varepsilon u^{9} \quad(u \geq 0) \quad \text { and } \quad f(u)=\alpha|u|^{r}+\delta|u|^{r} \quad(u \leq 0) .
$$

Then:

(i) There are constants $\Lambda>0$ and $\Lambda_{-}>0$ such that problem (1) has at least one sign-changing solution if $\lambda \leq \Lambda$, has no sign-changing solutions if $\lambda>\Lambda$, and the solution is unique if $\lambda<\Lambda_{-}$.

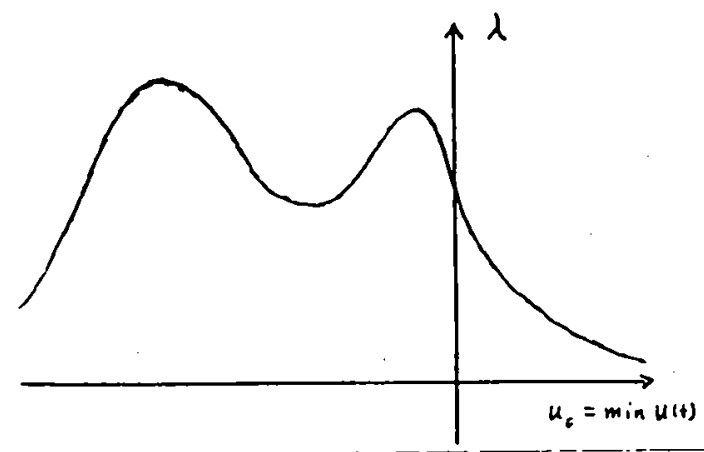

Figure 2 
(ii) For small $\varepsilon$ and $\delta$ satisfying $\varepsilon<<\delta$ there are $\lambda_{1}<\lambda_{2}$ such that if $\lambda \in\left(\lambda_{1}, \lambda_{2}\right)$, then problem (1) has at least four sign-changing solutions.

Next we consider for simplicity the semilinear $(p=2)$ problem

$$
\left.\begin{array}{l}
u^{\prime \prime}=\lambda f(u) \quad(a<t<b) \\
u(a)=u(b)=+\infty
\end{array}\right\}
$$

For this simple problem we have the following, a somehow surprising result.

Theorem 3. For given positive constants $\alpha, q$ and $\beta$ such that $q>3$ and $\beta<1$ let

$$
f(u)=\alpha u^{q} \quad(u \geq 0) \quad \text { and } \quad f(u)=(1+\beta \sin |u|)|u| \quad(u \leq 0)
$$

$\left(\lambda(2)=\left(\frac{\pi}{b-a}\right)^{2}\right)$. Then the following results hold:

(i) There are $\lambda_{1}, \lambda_{2}>0$ such that problem (2) has at least one sign-changing solution if $\lambda \geq \lambda_{1}$ and no sign-changing solutions if $\lambda<\lambda_{1}$. The sign-changing solution is unique, when $\lambda>\lambda_{2}$.

(ii) For any integer $n \geq 1$ there exists $\delta>0$ such that problem (2) has at least $n$ distinct sign-changing solutions when $\lambda \in(\lambda(2)-\delta, \lambda(2)+\delta)$.

(iii) For $\lambda=\lambda(2)$ problem (2) has infinitely many sign-changing solutions.

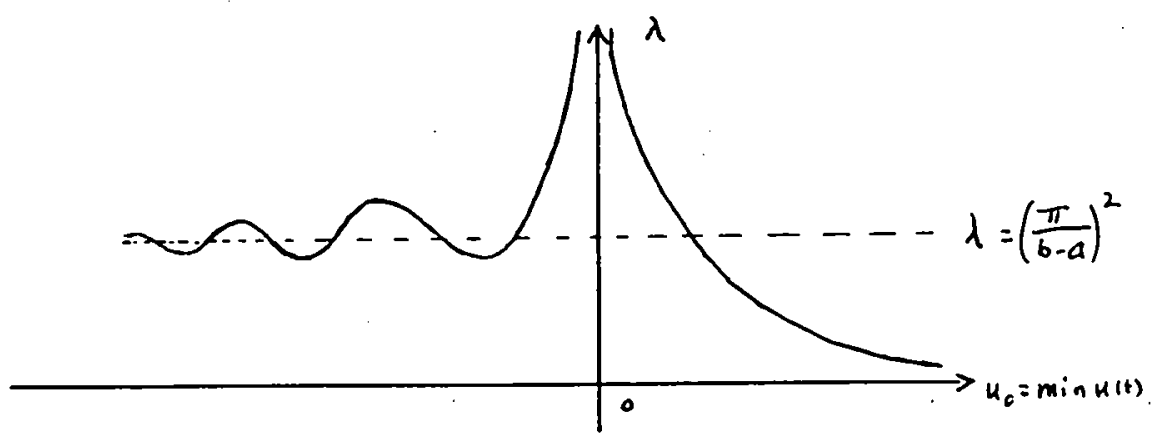

Figure 3

Remark. Problem (2) has a unique positive solution for all $\lambda>0$. The problems treated in Theorems 1 and 2 have also a unique positive solution if $\lambda<\lambda_{0}$ where $\lambda_{0}$ is defined by

$$
\int_{0}^{\infty} \frac{d u}{\sqrt{\frac{1}{q+1} u^{1+q}+\frac{\varepsilon}{s+1} u^{1+s}}}=\frac{b-a}{2} \sqrt[V]{p^{\prime} \lambda_{0}}
$$

and has no positive solution if $\lambda \geq \lambda_{0}$. 


\section{Some basic analysis}

It is easy to see that the equation in (1) has an first integral

$$
\frac{1}{p^{\prime}}\left|u^{\prime}(t)\right|^{p}=\lambda F(u)+C \quad \text { with } F(u)=\int_{0}^{u} f(x) d x
$$

where $\frac{1}{p}+\frac{1}{p^{\prime}}=1$. Let $t_{0} \in(a, b)$ be a minimum point of $u(t)$, which exists by the boundary condition. Then $u^{\prime}\left(t_{0}\right)=0$ and $C=-\lambda F\left(u_{0}\right), u_{0}=\min u(t)$, and

$$
\left|u^{\prime}(t)\right|^{p}=\lambda p^{\prime}\left(F(u)-F\left(u_{0}\right)\right) \quad(a<t<b) .
$$

If $f=f(u)$ is non-negative, then we see that $u=u(t)$ is convex and the minimum point $t=t_{0}$ is unique. Consequetly, $u^{\prime}(t) \leq 0$ for $t \in\left(a, t_{0}\right)$ and $u^{\prime}(t) \geq 0$ for $t \in\left(t_{0}, b\right)$. Moreover,

$$
\left.u^{\prime}(t)=\sqrt[8]{\lambda p^{\prime}\left(F(u)-F\left(u_{0}\right)\right.}\right) \operatorname{sign}\left(t-t_{0}\right) \quad(a<t<b) .
$$

Direct integration yields

$$
\left(b-t_{0}\right) \sqrt[R]{p^{\prime} \lambda}=\int_{u_{0}}^{+\infty} \frac{d u}{\sqrt[R]{F(u)-F\left(u_{0}\right)}}=\left(t_{0}-a\right) \sqrt[P]{p^{\prime} \lambda}
$$

which implies $t_{0}=\frac{a+b}{2}$ and thus $u=u(t)$ must be symmetric.

To establish the existence and the structure of solutions of problem (1) it suffices to study the nonlinear integral equation

$$
\int_{u_{0}}^{+\infty} \frac{d u}{\sqrt{F(u)-F\left(u_{0}\right)}}=\frac{b-a}{2} \sqrt[8]{p^{\prime} \lambda}
$$

Obviously, a necessary condition for the existence of solutions for problem (1) is

$$
\int^{+\infty} \frac{d u}{\sqrt{F(u)}}<+\infty
$$

and so throughout this paper we shall assume that this condition holds.

Rewriting the integral in (4) gives that it is equivalent to

$$
\int_{0}^{+\infty} \frac{d u}{\sqrt{F\left(u+u_{0}\right)-F\left(u_{0}\right)}}=\frac{b-a}{2} \sqrt[\nabla]{p^{\prime} \lambda}
$$

It follows from here that problem (1) has at most one (positive) solution under the condition that $f(u)$ is non-decreasing on $\mathbb{R}$ (or on $\mathbb{R}_{+}$). On the other hand, if $f(u)>0$ for $u>0$, then it has at least one positive solution for all $\lambda<\lambda_{0}$, where $\lambda_{0} \in(0,+\infty]$ is defined by

$$
\int_{0}^{+\infty} \frac{d u}{\sqrt{F(u)}}=\frac{b-a}{2} \sqrt[8]{p^{\prime} \lambda_{0}}
$$

By summarying, let $\lambda_{0}$ be as above. Then we have the following 
Theorem 4. If $f(u)>0 \quad(u>0)$, then problem (1) has at least one positive solution for all $\lambda<\lambda_{0}$. Moreover, if $f$ is also non-decreasing on $\mathbb{R}_{+}$, then the solution is unique.

Example. Consider the problem

$$
\left.\begin{array}{l}
\Delta_{p} u=\lambda\left(u^{q}+\varepsilon u^{s}\right) \quad(a<t<b) \\
u(a)=u(b)=+\infty
\end{array}\right\}
$$

where $0<r<q, \varepsilon>0$ and $q>p-1$. Then condition (5) is satisfied, and further $\lambda_{0}=+\infty$ if $s \geq p-1$ and $\lambda_{0}<+\infty$ if $s \in(0, p-1)$. Hence problem (8) has a unique positive solution for all $\lambda>0$ if $q>r \geq p-1$, and for all $\lambda<\lambda_{0}$ if $0<s<p-1$, where $\lambda_{0}$ satisfies

$$
\int_{0}^{+\infty} \frac{d u}{\sqrt[p]{\frac{1}{q+1} u^{1+q}+\frac{e}{s+1} u^{1+s}}}=\frac{b-a}{2} \sqrt[p]{p^{\prime} \lambda_{0}} .
$$

\section{Proofs}

To investigate solutions of problem (1) which change its sign, we define

$$
f_{+}(u)=f(u)(u \geq 0) \quad \text { and } \quad f_{-}(u)=f(-u)(u \leq 0) .
$$

Then we have

$$
F(u)= \begin{cases}F_{+}(u)=\int_{0}^{u} f_{+}(x) d x & \text { for } u \geq 0 \\ -F_{-}(-u)=-\int_{0}^{-u} f_{-}(x) d x & \text { for } u<0 .\end{cases}
$$

Now equation (6) becomes

$$
\int_{0}^{+\infty} \frac{d u}{\sqrt{F_{+}(u)+F_{-}\left(v_{0}\right)}}+\int_{0}^{v_{0}} \frac{d u}{\sqrt{F_{-}\left(v_{0}\right)-F_{-}(u)}}=\frac{b-a}{2} \sqrt{p^{\prime} \lambda}
$$

where $v_{0}=-u_{0}>0$. First observe that the first integral in (9) is strictly decreasing in $v_{0}$ if $f_{-}(u)$ is non-negative. For the second integral in (9), using

$$
\int_{0}^{v_{0}} \frac{d u}{\sqrt{F_{-}\left(v_{0}\right)-F_{-}(u)}}=\int_{0}^{1} \frac{v_{0} d s}{\sqrt{F_{-}\left(v_{0}\right)-F_{-}\left(s v_{0}\right)}}
$$

and

$$
F_{-}\left(v_{0}\right)-F_{-}\left(s v_{0}\right)=\int_{0}^{1} f_{-}\left((s+t-s t) v_{0}\right)(1-s) v_{0} d t
$$


we see that it is decreasing or increasing in $v_{0}$ if $f_{-}(u) u^{1-p}$ is increasing or decreasing, respectively: Therefore, if $f_{-}(u)$ is non-negative and $f_{-}(u) u^{1-p}$ is increasing, then (9) has at most one solution, which gives the uniqueness of sign-changing solutions of problem (1). On the other hand, if $f_{-}(u) u^{1-p}$ is decreasing, then the first and the second integrals in (9) will compete to each other and thus the existence of a multiple solution is possible. In particular, if

$$
\int_{0} \frac{d u}{\sqrt{F_{+}(u)}}=+\infty \quad \text { and } \quad \lim _{u \rightarrow+\infty} f_{-}(u) u^{1-p}=0
$$

then the left side in (9) goes to infinity, when $v_{0}$ goes either to zero or to infinity. Hence there is $\lambda_{-}>0$ such that problem (1) has at least two sign-changing solutions for $\lambda>\lambda_{-}$, and no sign-changing solution if $\lambda<\lambda_{-}$. A typical example is

$$
f_{+}(u)=\sum \alpha_{i} u^{q_{i}} \quad \text { and } \quad f_{-}(u)=\sum \beta_{j} u^{r_{j}}
$$

where $\alpha_{i}, \beta_{j}>0$ and $q_{i}>p-1>r_{j}$. When $f_{+}$and $f_{-}$are simply given by $\alpha u^{q}$ and $\beta u^{r} \quad(q>p-1>r)$, respectively, then we have a complete charaterization of signchanging solutions, namely, two solutions if $\lambda>\lambda_{-}$, a unique solution if $\lambda=\lambda_{-}$, and no solutions if $\lambda<\lambda_{-}$. In other situations the multiplicity of sign-changing solutions can be very complicated and three representive examples are as in Theorems 1 - 3 .

Proof of Theorem 1. To this end we study the function in the left-hand side of (9). In this case we have

$$
F_{+}(u)=\frac{1}{q+1} u^{q+1}+\frac{\varepsilon}{1+s} u^{1+s} \quad \text { and } \quad F_{-}(u)=\frac{\alpha}{r+1} u^{r+1}
$$

and

$$
\int_{0}^{1} \frac{v_{0} d s}{\sqrt{F_{-}\left(v_{0}\right)-F_{-}\left(s v_{0}\right)}}=c_{2} v_{0}^{1-\frac{1+r}{p}} \quad \text { with } \quad c_{2}=\int_{0}^{1} \frac{d s}{\sqrt[8]{\alpha\left(1-s^{r+1}\right) /(1+r)}}
$$

and the function in (9) is

$$
\int_{0}^{\infty} \frac{d u}{\sqrt{\frac{1}{q+1} u^{q+1}+\frac{\varepsilon}{1+s} u^{1+s}+\frac{\alpha}{1+r} v_{0}^{r+1}}}+c_{2} v_{0}^{1-\frac{r+1}{p}}=: F_{\varepsilon}\left(v_{0}\right)
$$

The first conclusion of Theorem 1 follows from that $F_{e}\left(v_{0}\right)>0$ is continuous on $[0,+\infty)$ and goes to infinity, as $v_{0} \rightarrow \infty$. To get a complete picture for the existence of signchanging solutions we let

$$
k_{1}=(1+r)\left(\frac{1}{s+1}-\frac{1}{p}\right), \quad k_{2}=\frac{(1+r)(q-s)}{1+s}, \quad k_{3}=1-\frac{r+1}{p}
$$

and

$$
g\left(v_{0}\right)=\int_{0}^{+\infty}\left(\frac{1}{q-1} u^{q+1}+\frac{\varepsilon}{1+s^{-1}} u^{1+s}+\frac{\alpha}{1+r} v_{0}^{r+1}\right)^{-\frac{1}{p}} d u
$$


Then $F_{e}\left(v_{0}\right)=g\left(v_{0}\right)+c_{2} v_{0}^{k_{3}}$. We first study the property of $g\left(v_{0}\right)$ in a neighbourhood of the origin.

By the change of variable $u=x v_{0}^{\frac{1+5}{1+\frac{1}{2}}}$ we get for $v_{0}>0$

$$
\begin{aligned}
g\left(v_{0}\right) & =\int_{0}^{+\infty} \frac{v_{0}^{k_{1}} d x}{\sqrt{\frac{1}{q+1} x^{q+1} v_{0}^{k_{2}}+\frac{\varepsilon}{1+s} x^{1+s}+\frac{\alpha}{1+r}}} \\
g(0) & =\int_{0}^{+\infty} \frac{v_{0}^{k_{1}} d x}{\sqrt{\frac{1}{q+1} x^{q+1} v_{0}^{k_{2}}+\frac{\varepsilon}{1+s} x^{1+s}}} .
\end{aligned}
$$

Consequently,

$$
\frac{g\left(v_{0}\right)-g(0)}{v_{0}^{k_{1}}}=-\int_{0}^{+\infty} G\left(v_{0}, x\right) d x
$$

where

$$
G\left(v_{0}, x\right)=\frac{1}{\sqrt{\frac{1}{q+1} x^{q+1} v_{0}^{k_{2}}+\frac{\varepsilon}{1+s} x^{1+s}}}-\frac{1}{\sqrt{\frac{1}{q+1} x^{q+1} v_{0}^{k_{2}}+\frac{\varepsilon}{1+s} x^{1+s}+\frac{\alpha}{1+r}}} .
$$

Writing the difference as an integral we see

$$
G\left(v_{0}, x\right)=\frac{\alpha}{p(r+1)} \int_{0}^{1}\left(\frac{1}{q+1} x^{q+1} v_{0}^{k_{2}}+\frac{\varepsilon}{1+s} x^{1+s}+\frac{\alpha}{1+r} \theta\right)^{-\frac{q+1}{p}} d \theta
$$

which implies that $G\left(v_{0}, x\right)$ is decreasing in $v_{0}$. Since

$$
G(0, x)=\frac{\alpha}{p(r+1)} \int_{0}^{1}\left(\frac{\varepsilon}{1+s} x^{1+s}+\frac{\alpha}{1+r} \theta\right)^{-\frac{R+1}{p}} d \theta
$$

is integrable over $(0,+\infty)$, due to $s \in(0, p-1)$, we deduce by the dominate convergence theorem that

$$
\lim _{v_{0} \rightarrow 0} \frac{g\left(v_{0}\right)-g(0)}{v_{0}^{k_{1}}}=-\int_{0}^{+\infty} G(0, x) d x<0 .
$$

Thus we obtain that near the origin $F_{\varepsilon}$ is increasing if $k_{1}>1-\frac{r+1}{p}$, which is equivalent to $r>s$, and is decreasing when $r<s$. Similarly, we get that

$$
\lim _{v_{0} \rightarrow \infty} g^{\prime}\left(v_{0}\right) v_{0}^{1+(r+1)\left(\frac{1}{p}-\frac{1}{q+1}\right)}=-\frac{\alpha}{p} \int_{0}^{1}\left(\frac{1}{q+1} x^{q+1}+\frac{\alpha}{1+r}\right)^{-\frac{p+1}{p}} d x
$$


which implies that $F_{e}$ is increasing for large $v_{0}>0$, because $q>p-1$. Since $\frac{d F_{0}}{d v_{0}}$ is increasing in $\varepsilon$ and tends to $\left(1-\frac{r+1}{p}\right) c_{2} v_{0}^{-\frac{r+1}{p}}$ uniformly in $v_{0}$ as $\varepsilon \rightarrow \infty$, we deduce in the case $r>s$ that there is an $\varepsilon_{0} \geq 0$ such that $\min _{v_{0}} \frac{d F_{v}}{d v_{0}}>0$ for $\varepsilon>\varepsilon_{0}$. Moreover, when $\varepsilon=0$,

$$
F_{0}\left(v_{0}\right)=c_{1} v_{0}^{1-\frac{e+1}{p}}+c_{2} v_{0}^{1-\frac{r+1}{p}}
$$

for $1-\frac{q+1}{p}<0<1-\frac{r+1}{p}$ and thus $\frac{d F_{0}}{d v_{0}}<0$ for small $v_{0}>0$. Further, $F_{\varepsilon}\left(v_{0}\right) \rightarrow F_{0}\left(v_{0}\right)$ on compacta in $C^{1}(0,+\infty)$ as $\varepsilon \rightarrow 0$ and we assert that $\varepsilon_{0}>0$ and $\min _{v_{0}} \frac{d F_{\varepsilon}}{d v_{0}}<0$ for $\varepsilon \in\left(0, \varepsilon_{0}\right)$. Whence, we obtain that the sign-changing solution is unique when $\varepsilon \geq \varepsilon_{0}$. If $\varepsilon \in\left(0, \varepsilon_{0}\right)$, using the fact that $F_{\varepsilon}$ is increasing for both small and large $v_{0}$, we deduce that there are $0<v_{1}<v_{2} \leq v_{3}<+\infty$ such that $F_{\varepsilon}$ is increasing on $\left(0, v_{1}\right)$ and $\left(v_{3},+\infty\right)$ and is decreasing on $\left(v_{1}, v_{2}\right)$. Thus let

$$
\lambda_{1}=\min F_{\varepsilon}\left(v_{0}\right), \quad \lambda_{2}=F_{\varepsilon}\left(v_{1}\right), \quad \lambda_{3}=F_{\varepsilon}\left(v_{2}\right), \quad \lambda_{4}=\max _{F_{\varepsilon}^{\prime}\left(v_{0}\right)=0} F_{\varepsilon}\left(v_{0}\right) .
$$

Then problem (1) has no sign-changing solutions if $\lambda<\lambda_{1}$ and has at least two signchanging solutions $\lambda \in\left(\lambda_{1}, \lambda_{4}\right)$. In particular, it has at least three sign-changing solutions if $\lambda \in\left(\lambda_{2}, \lambda_{3}\right)$ and has a unique sign-changing solution as $\lambda>\lambda_{4}$. The proof is complete

Proof of Theorem 2. The idea is the same as in the proof of Theorem 1, so we will be sketch in many places. In view of

$$
F_{+}(u)=\frac{1}{q+1} u^{q+1}+\frac{\varepsilon}{1+s} u^{1+s} \quad \text { and } \quad F_{-}(u)=\frac{\alpha}{r+1} u^{r+1}+\frac{\delta}{\tau+1} u^{r+1}
$$

it follows that equation (9) takes the form

$$
\begin{aligned}
& \int_{0}^{+\infty} \frac{d u}{\sqrt{\frac{1}{q+1} u^{q+1}+\frac{\varepsilon}{1+s} u^{1+s}+F_{-}\left(v_{0}\right)}} \\
& \quad+\int_{0}^{1} \frac{v_{0} d x}{\sqrt{r(x) v_{0}^{s+1}+\tau(x) v_{0}^{\tau+1}}}=\frac{b-a}{2} \sqrt[R]{\alpha p^{\prime} \lambda}
\end{aligned}
$$

where

$$
r(x)=\frac{\alpha}{r+1}\left(1-x^{r+1}\right) \quad \text { and } \quad \tau(x)=\frac{\delta}{\tau+1}\left(1-x^{r+1}\right) .
$$

Likely as in the proof of Theorem 1 we define

$$
L\left(v_{0}\right)=\ell_{1}\left(v_{0}\right)+\ell_{2}\left(v_{0}\right)
$$

where

$$
\begin{aligned}
& \ell_{1}\left(v_{0}\right)=\int_{0}^{+\infty} \frac{d u}{\sqrt{\frac{1}{q+1} u^{q+1}+\frac{\varepsilon}{1+s} u^{1+s}+F_{-}\left(v_{0}\right)}} \\
& \ell_{2}\left(v_{0}\right)=\int_{0}^{1} \frac{v_{0} d x}{\sqrt[p]{r(x) v_{0}^{r+1}+\tau(x) v_{0}^{r+1}}}
\end{aligned}
$$


The key points to prove that the function $L\left(v_{0}\right)$ have a graph as shown in Figure 2 are

$$
L\left(v_{0}\right)=F_{0}\left(v_{0}\right)=c_{1} v_{0}^{p-q-1}+c_{2} v_{0}^{p-r-1} \quad \text { when } \varepsilon=\delta=0
$$

which has the property $F_{0}^{\prime}\left(v_{0}\right)\left(v_{0}-v\right)>0$ for some $v>0$ and $L$ converges to $F_{0}$ on compacta of $(0,+\infty)$ in $C^{1}$ as $\varepsilon, \delta \rightarrow 0$ and $L \rightarrow 0$ as $v_{0} \rightarrow+\infty$.

To complete the proof we need to show two more things: Monotonicity of $L$ near the origin and $L(0) \leq L\left(v_{2}\right)$ where $v_{2}$ is the second local maximum of $L$. But

$$
L(0)=c_{1} \varepsilon^{\frac{q-p-1}{p(q-1)}} \quad \text { and } \quad L\left(\delta^{\frac{1}{r-r}}\right) \geq \ell_{2}\left(\delta^{\frac{1}{r-r}}\right)=c_{2} \delta^{\frac{r+1-p}{p(r-r)}}
$$

for some constants $c_{1}>0$ and $c_{2}>0$ from which it follows that if $\varepsilon$ and $\delta$ satisfy $c_{1} \varepsilon^{\frac{q-p-1}{p(q-r)}} \leq c_{2} \delta^{\frac{r+1-p}{p(r-r)}}$, then we are done.

To get the monotonicity of $L$ in the nearby of the origin, we exploit the technique in the proof of Theorem 1 and can show that

$$
\lim _{v_{0} \rightarrow 0} \frac{\ell_{1}\left(v_{0}\right)-\ell_{1}(0)}{v_{0}^{k_{1}}}=-\frac{\alpha}{p(r+1)} \int_{0}^{+\infty} d x \int_{0}^{1}\left(\frac{\varepsilon}{1+s} x^{s+1}+\frac{\alpha}{r+1} \theta\right)^{-\frac{p+1}{p}} d \theta<0
$$

where we have used that $r>\tau$ and $F_{-}\left(v_{0}\right)=\frac{\alpha}{r+1} v_{0}^{r+1}(1+o(1))$ as $v_{0} \rightarrow 0$,

$$
\lim _{v_{0} \rightarrow 0} \frac{\ell_{2}\left(v_{0}\right)-\ell_{2}(0)}{v_{0}^{k_{3}}}=\int_{0}^{1} \frac{d x}{\sqrt{r(x)}}>0 . \quad \text { and } \quad \lim _{v_{0} \rightarrow 0} \frac{L\left(v_{0}\right)-L(0)}{v_{0}^{k_{1}}}<0 .
$$

Finally, the uniqueness follows from the results in [4] that the function $\ell_{2}$ has the following property: there is $v>0$ such that $\ell_{2}^{\prime}\left(v_{0}\right)>0$ for $v_{0} \in(0, v)$ and $\ell_{2}^{\prime}\left(v_{0}\right)<0$ for $v_{0} \in(v,+\infty)$. Then $L^{\prime}\left(v_{0}\right)<0$ for $v_{0} \in[v,+\infty)$ since clearly $\ell_{1}^{\prime}\left(v_{0}\right)<0$ for $v_{0}>0$. The proof is complete

Proof of Theorem 3. By virtue of $p=2$ and

$$
F_{+}(u)=\frac{\alpha}{q+1} u^{q+1} \quad \text { and } \quad F_{-}(u)=\frac{1}{2} u^{2}+\beta(\sin u-u \cos u)
$$

we have that

$$
\begin{gathered}
\int_{0}^{+\infty} \frac{d u}{\sqrt{F_{+}(u)+F_{-}\left(v_{0}\right)}}=c(q)\left(\frac{1}{2} v_{0}^{2}+\beta\left(\sin v_{0}-v_{0} \cos v_{0}\right)\right)^{\frac{1}{i+1}-\frac{1}{2}}=: g_{1}\left(v_{0}\right) \\
\int_{0}^{v_{0}} \frac{d u}{\sqrt{F_{-}\left(v_{0}\right)-F_{-}(u)}}=\int_{0}^{1} \frac{v_{0} d x}{\sqrt{h\left(x, v_{0}\right)}}=: g_{2}\left(v_{0}\right)
\end{gathered}
$$


where

$$
\begin{aligned}
h\left(x, v_{0}\right) & =e(x) v_{0}^{2}+\beta \varepsilon\left(x, v_{0}\right) \\
\varepsilon\left(x, v_{0}\right) & =\sin v_{0}-\sin \left(x v_{0}\right)-v_{0}\left(\cos v_{0}-x \cos \left(x v_{0}\right)\right) \\
c(q) & =\int_{0}^{+\infty}\left(1+\frac{\alpha}{q+1} u^{q+1}\right)^{-\frac{1}{2}} d u \\
e(x) & =\frac{1-x^{2}}{2}
\end{aligned}
$$

and the function in the left-hand side of (9) takes the form

$$
g_{1}\left(v_{0}\right)+g_{2}\left(v_{0}\right):=H\left(v_{0}\right) \text {. }
$$

Clearly, $H$ is well defined and continuous on $(0,+\infty)$. Since $q>3$, we see that

$$
g_{1}\left(v_{0}\right) \rightarrow+\infty \quad \text { as } \quad v_{0} \rightarrow 0+
$$

and

$$
\left.\begin{array}{l}
g_{1}\left(v_{0}\right) \rightarrow 0 \\
g_{2}\left(v_{0}\right) \rightarrow \int_{0}^{1} \frac{d x}{\sqrt{e(x)}}=\frac{\pi}{\sqrt{2}}
\end{array}\right\} \quad \text { as } v_{0} \rightarrow+\infty
$$

and therefore $H(0+)=+\infty$ and $H(+\infty)=\frac{\pi}{\sqrt{2}}$.

To show the results (ii) and (iii) we need to prove that $H$ oscillates around the line $H=\frac{\pi}{\sqrt{2}}$, and first show that $g_{1}$ oscillates around $H=\frac{\pi}{\sqrt{2}}$.

Choosing $v_{0}=n \pi \quad(n \in \mathbb{N}$ is even) we get

$$
\begin{aligned}
h(x, n \pi) & =(n \pi)^{2} e(x)-\beta(\sin (n \pi x)+n \pi(1-x \cos (n \pi x))) \\
& \leq(n \pi)^{2} e(x)-\beta(n \pi(1-x)-\sin (n \pi(1-x))) \\
& \leq(n \pi)^{2} e(x)
\end{aligned}
$$

thereafter

$$
g_{2}(n \pi)>\int_{0}^{1} \frac{n \pi d x}{\sqrt{e(x)(n \pi)^{2}}}=\frac{\pi}{\sqrt{2}} .
$$

Analogously, for odd integer $n, g_{2}(n \pi)<\frac{\pi}{\sqrt{2}}$.

To show the function $H$ is also oscillating, it suffices to show that, for odd $n$, $H(n \pi)<\frac{\pi}{\sqrt{2}}$ since $g_{1}>0$ and $g_{2}(n \pi)>\frac{\pi}{\sqrt{2}}$ for even $n$. For this purpose, first we have

$$
\begin{aligned}
\frac{\pi}{\sqrt{2}}-g_{2}(n \pi) & =\int_{0}^{1}\left(\frac{n \pi}{\sqrt{e(x)(n \pi)^{2}}}-\frac{n \pi}{\sqrt{e(x)(n \pi)^{2}+\beta \varepsilon(x, n \pi)}}\right) d x \\
& =\frac{1}{2} \int_{0}^{1} d x \int_{0}^{1} \frac{n \pi \beta \varepsilon(x, n \pi)}{\left(e(x)(n \pi)^{2}+\theta \beta \varepsilon(x, n \pi)\right)^{\frac{3}{2}}} d \theta \\
& \geq \frac{1}{2} \int_{0}^{1} \frac{n \pi \beta \varepsilon(x, n \pi)}{\left(e \sqrt{(x)(n \pi)^{2}}+\beta \varepsilon(x, n \pi)\right)^{\frac{3}{2}}} d x, \ldots-\ldots
\end{aligned}
$$


due to $\varepsilon(x, n \pi)>0$ for all $x \in(0,1)$. Using $f_{-}(u) \geq(1-\beta) u$, we deduce that

$$
\begin{aligned}
e(x)(n \pi)^{2}+\beta \varepsilon(x, n \pi) & =n \pi(1-x) \int_{0}^{1} f_{-}((x+t-t x) n \pi) d t \\
& \geq n \pi(1-x) \int_{0}^{1}(1-\beta)(x+t-t x) n \pi d t \\
& \geq \frac{1}{2}(1-\beta)(1-x)(n \pi)^{2}
\end{aligned}
$$

for all $x \in(0,1)$. Thereafter

$$
\frac{\pi}{\sqrt{2}}-g_{2}(n \pi) \geq \frac{2^{\frac{3}{2}} \beta}{(1-\beta)^{\frac{3}{2}}}(n \pi)^{-2} \int_{0}^{1} \frac{\varepsilon(x, n \pi)}{(1-x)^{\frac{3}{2}}} d x,
$$

and by a change of variable $z=\frac{1}{\sqrt{1-x}}\left(\right.$ then $\left.x=1-\frac{1}{z^{2}}\right)$

$$
\begin{aligned}
\int_{0}^{1} \frac{\varepsilon(x, n \pi)}{(1-x)^{\frac{3}{2}}} d x & =2 \int_{1}^{+\infty} \varepsilon\left(1-\frac{1}{z^{2}}, n \pi\right) d z \\
& =2 \int_{1}^{+\infty}\left(-\sin \left(\frac{n \pi}{z^{2}}\right)+n \pi\left(1-\left(1-\frac{1}{z^{2}}\right) \cos \frac{n \pi}{z^{2}}\right)\right) d z c r \\
& =2 \int_{1}^{+\infty}\left(n \pi\left(1-\cos \frac{n \pi}{z^{2}}\right)+\left(-\sin \frac{n \pi}{z^{2}}+\frac{n \pi}{z^{2}} \cos \frac{n \pi}{z^{2}}\right)\right) d z
\end{aligned}
$$

where we have used $n$ being an odd integer. Change variables once more, we obtain

$$
\int_{1}^{+\infty}\left(1-\cos \frac{n \pi}{z^{2}}\right) d z=\int_{\frac{1}{\sqrt{n \pi}}}^{+\infty}\left(1-\cos \frac{1}{w^{2}}\right) \sqrt{n \pi} d w \geq C \sqrt{n \pi}
$$

where $C=\int_{1}^{+\infty}\left(1-\cos \frac{1}{z^{2}}\right) d z$. On the other hand,

$$
\int_{1}^{+\infty}\left(-\sin \frac{n \pi}{z^{2}}+\frac{n \pi}{z^{2}} \cos \frac{n \pi}{z^{2}}\right) d z \leq \int_{1}^{+\infty} \frac{2 n \pi}{z^{2}} d z=2 n \pi .
$$

Whence

$$
\int_{0}^{1} \frac{\varepsilon(x, n \pi)}{(1-x)^{\frac{3}{2}}} d x \geq 2(C, n \pi \sqrt{n \pi}-2 n \pi)
$$


and consequetly for large $n$ the estimate

$$
\frac{\pi}{\sqrt{2}}-g_{2}(n \pi) \geq \frac{2^{\frac{3}{2}} \beta}{(1-\beta)^{\frac{3}{2}}} C(n \pi)^{-\frac{1}{2}}
$$

holds. Combing the above estimates, we obtain by noting $g_{1}(n \pi) \leq C_{1}(n \pi)^{\frac{2}{a+1}-1}$ that

$$
\frac{\pi}{\sqrt{2}}-H(n \pi)=\frac{\pi}{\sqrt{2}}-g_{2}(n \pi)-g_{1}(n \pi) \geq C_{2}(n \pi)^{-\frac{1}{2}}-C_{1}(n \pi)^{\frac{2}{\sigma+1}-1}>0
$$

since $-\frac{1}{2}>\frac{2}{q+1}-1$ (this is the source for the condition on $q$ ).

Assertions (ii) and (iii) follow from the oscillatory property of $H$. Using

$$
\lim _{v_{0} \rightarrow \infty} H\left(v_{0}\right)=\frac{\pi}{\sqrt{2}}, \quad \lim _{v_{0} \rightarrow 0+} H\left(v_{0}\right)=+\infty, \quad H(n \pi)<\frac{\pi}{\sqrt{2}}
$$

we deduce from the continuity of $H$ that the minimum of $H\left(v_{0}\right)$ on $(0,+\infty)$ achives and thus the equation $H\left(v_{0}\right)=\mu$ is solvable if and only, if $\mu \geq \min H\left(v_{0}\right)$ which complete the proof of assertion (i)

\section{Final remarks}

In this note we have only carried out some basic calculations to exhibit the rich structure for boundary blow-up problems, even it is very elementary (just calculus), but it is certainly not easy to give a complete bifurcation picture for all involved parameters, for instance $q, r, s, \tau, \varepsilon, \delta, \alpha$ in Theorem 2. From our one-dimensional examples we can see that there is a big difference between Dirichlet boundary value problems and boundary bolw-up problems. If the boundary is Dirichlet, then there are infinitely many sign solutions in the superlinear case, but it can have only finite number of sign solutions for boundary bolw-up problems (note, the function in (2) is not superlinear at $-\infty$ ). As our examples are of one-dimensional character, one may say that it would not be representive, so it will be interesting to study those equations in two-dimensional or higher dimensional domains.

\section{References}

[1] Aftalion, A. and W. Reichel: Existence of two boundary blow-up solutions for semilinear elliptic equations. J. Diff. Equ. 141 (1997), $400-421$.

[2] Bandle, C. and M. Essén: On the solutions of quasilinear elliptic problems with boundary blow-up. In: Partial Differential Equations of Elliptic Type (Symposia Mathematica: Vol. 35; eds.: A. Alvino at al.). Univ. Press, Cambridge (1994), pp. $93-111$.

[3] Bandle, C. and M. Marcus: Asymptotic behaviour of solutions and their derivatives, for semilinear elliptic problems_with_boundary-blow-up.-Ann-Inst--Henri-Poicaré-12-(1995), $155-171$. 
[4] Cheng, Y. J.: On an open problem of Ambrosetti, Brezis and Cerami. Diff. Int. Equ. (to appear).

[5] Keller, J. B.: Electrohydrodynamics. Part I: The equilibrium of a charged gas in a container. J. Rat. Mech. Anal. 5 (1956), 715 - 724.

[6] Loewner, C. and L. Nirenberg: Partial differential equations invariant under conformal or projective transformations. In: Contribution to Analysis (ed.: L. Ahlfors). Acad. Press, New York (1974), pp. $245-272$.

[7] Matero, J.: Nonlinear Elliptic Problems with Bouindary Blow-Up. Diss. in Math. 6. Uppsala: Univ. 1997.

[8] Mckenna, P. J., Reichel, W. and W. Walter: Symmetry and multiplicity for nonlinear elliptic differential equations with boundary blow-up. Nonlin. Anal. Theory, Methods and Appl. 28 (1997), 1213 - 1225.

Received 26.10.1998; in revised form 21.04.1999 